\title{
Diagnóstico de prácticas de iniciación y planeación en gerencia de proyectos en pymes del sector de la construcción ${ }^{1}$
}

Germán Eduardo Giraldo González ${ }^{2}$ Escuela Colombiana de Ingeniería Julio Garavito german.giraldo@escuelaing.edu.co

Juan Carlos Castañeda Mondragón ${ }^{3}$ Escuela Colombiana de Ingeniería Julio Garavito juan.castaneda-m@mail.escuelaing.edu.co

Orlando Correa Basto ${ }^{4}$

Fecha de recepción: 19 de julio de 2017

Fecha de aprobación: 22 de septiembre de 2017

DOI: https://doi.org/10.21158/01208160.n0.2018.2018
Escuela Colombiana de Ingeniería Julio Garavito orlando.correabasto@gmail.com

Juan Carlos Sánchez Ángel ${ }^{5}$ Escuela Colombiana de Ingeniería Julio Garavito Ingjuancarlos_86@hotmail.com

Cómo citar este artículo / To reference this article / Comment citer cet article / Para citar este artigo:

Giraldo González, G. E.; Castañeda Mondragón, J. C.; Correa Basto, O. y Sánchez Ángel, J. C. (2018). Diagnóstico de prácticas de iniciación y planeación en gerencia de proyectos en pymes del sector de la construcción. Revista EAN, Edición especial, pp.55-83. DOI: https://doi.org/10.21158/01208160.n0.2018.2018

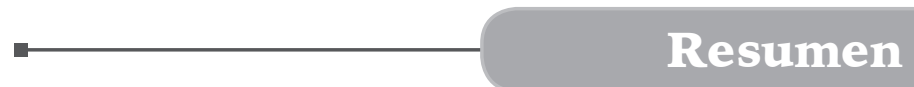

El presente artículo presenta los resultados del diagnóstico de las prácticas de iniciación y planificación en gerencia de proyectos para pymes del sector de la construcción en Bogotá, Colombia. Esta investigación se basó en el estudio de la bibliografía internacional relacionada con las pymes, la gerencia de proyectos en el sector de la construcción y los procesos de iniciación y planificación. La información y los hallazgos se compararon con un conjunto de entrevistas y encuestas realizadas a gerentes de proyectos con experiencia relevante en la materia, lo que dio como resultado una caracterización de las prácticas en gerencia de proyectos que se aplican actualmente en las pymes del sector de la construcción. La descripción se realizó mediante la división de los hallazgos en las áreas de conocimiento establecidas en la guía del PMBOK ${ }^{\circledR}$ y la extensión para proyectos de construcción, para finalmente determinar las variables que pueden usarse en un futuro modelo para la gerencia de proyectos en pymes.

\section{Palabras Clave}

Dirección de proyectos, planificación del desarrollo, pymes - Colombia, diseño de proyectos, industria de la construcción.

\footnotetext{
${ }^{1}$ Los autores expresan su agradecimiento a la Unidad de Proyectos de la Escuela Colombiana de Ingeniería Julio Garavito, en especial al ingeniero César Leal; a los jurados y gerentes participantes en la investigación; a Javier Ramírez, quien nos ayudó con los instrumentos; al igual que a los asesores y profesores de la Maestría en Desarrollo y Gerencia Integral de Proyectos, por sus valiosos aportes y permanente apoyo.

${ }^{2}$ Ingeniero Industrial con Maestría en Ciencias en Ingeniería Industrial, Project Management Professional PMP® (PMI) y Auditor Interno en Sistemas de Gestión de la Calidad ISO 9001. Líder de Investigación e Innovación, gerente de proyectos de investigación y director de la Revista en Desarrollo y Gerencia Integral de Proyectos de la Unidad de Proyectos de la Escuela Colombiana de Ingeniería Julio Garavito. ORCID: http://orcid.org/0000-0002-2978-6049

${ }^{3}$ Economista de la Escuela Colombiana de Ingeniería, Mágister en Gerencia Integral de Proyectos de la misma universidad. Actualmente afiliado al área de Control Operacional y Proyectos de Avianca. ORCID: https://orcid.org/0000-0002-21992964

${ }^{4}$ Ingeniero Ambiental con Maestría en Gerencia Integral de Proyectos de la Escuela Colombiana de Ingeniería Julio Garavito. Gerente General y Representante Legal de Ingeniería Correa Correa S.A.S. ORCID: https://orcid.org/0000-0003-25950216

${ }^{5}$ Ingeniero Civil con Maestría en Gerencia Integral de Proyectos de la Escuela Colombiana de Ingeniería Julio Garavito. Director de Compras y Contratos en la empresa Hitos Urbanos. Participante de la Unidad de Proyectos de la Escuela Colombiana de Ingeniería Julio Garavito.
} 


\title{
Diagnosis of initiation and planning of project management in pymes from the construction economic sector
}

\begin{abstract}
This paper describes the outcomes of the diagnosis of initiation and planning stages in project management of PyMES from the construction economic sector in Bogota. This study is focused on international bibliographic revision about PyMES, construction project management and initiation and planning. Its information and findings are compared with some interviews and surveys made to well-experienced project managers, which resulted in the description of project management processes being currently applied in PyMES of the construction sector. This description was made by dividing the findings in the related knowledge areas included in PMBOK $®$ guidelines and the duration of construction projects to finally determine all possible variables that could be applied in project management future models in PyMES.
\end{abstract}

key words: Project management, developmental planning, PyMES- Colombia, project design, construction economic sector

\section{Diagnostic des pratiques d'initiation et de planification de gestion de projet des PME du secteur de la construction}

Résumé. Cet article présente les résultats du diagnostic des pratiques d'initiation et de planification en gestion de projet des PME du secteur de la construction de Bogotá, en Colombie. Cette investigation se base sur l'étude de la bibliographie internationale relative aux PME, la gestion de projets dans le secteur de la construction et les processus d'initiation et de planification. Les informations et les résultats obtenus ont été comparés à une série d'entretiens et d'enquètes réalisés auprès de gestionnaires de projet possédant une expérience pertinente dans le domaine et donnant lieu à une caractérisation des pratiques pour la gestion des projets en cours dans les PME du secteur de la construction. Cette description a été réalisée en prenant en compte les résultats des domaines de connaissances établies dans le guide $P M B O K{ }^{\circledR}$ et leur extension aux projets de construction afin de déterminer les variables pouvant être utilisées lors d'un futur modèle de gestion de projets pour les PME.

Mots clefs: Gestion de projet, planification du développement, PME - Colombie, conception de projets, industrie de la construction.

\section{Diagnóstico de práticas de iniciação e planejamento em gerência de projetos em MPEs do setor da construção}

Resumo. O presente artigo apresenta os resultados do diagnóstico das práticas de iniciação e planejamento em gerencia de projetos para as micro e pequenas empresas (MPEs) do setor da construção em Bogotá, Colômbia. Esta pesquisa baseou-se no estudo da bibliografia internacional relacionada com as MPEs, com a gerência de projetos no setor da construção e com os processos de iniciação e planejamento. A informação e os resultados compararamse com um conjunto de entrevistas e questionários realizados a gerentes de projetos com experiência relevante na matéria, o que deu como resultado uma caracterização das práticas em gerência de projetos que se aplicam atualmente nas MPEs do setor da construção. A descrição realizou-se mediante a divisão dos resultados nas áreas de conhecimento estabelecidas na guia do PMBOK ${ }^{\circledR}$ e a extensão para projetos de construção, para finalmente determinar as variáveis que podem ser usadas num futuro modelo para a gerência de projetos em MPEs.

Palavras-chave: Direção de projetos, planejamento do desenvolvimento, MPEs - Colômbia, desenho de projetos, indústria da construção. 


\section{Introducción}

$\mathrm{M}^{3}$ ás del $95 \%$ de las empresas del sector de la construcción en el país están clasificadas como pymes y cerca de la mitad se encuentran en Bogotá (Vega, Castaño y Mora, 2011). En el estudio realizado por Vargas (2015), se evidenciaron falencias en la planeación de proyectos, lo que arroja desviaciones en el alcance, el tiempo, el costo y la calidad. La mayoría de las organizaciones toman poco tiempo en la planeación y es frecuente que esta etapa la realicen durante la ejecución del proyecto. Faniran, Oluwoye y Lenard (1994) concluyen que el tiempo invertido en la planeación se ve bien recompensado en el éxito del proyecto, mientras que Elonen y Artto (2003) identifican la definición y planeación inadecuada como una de las causas de los problemas de los proyectos.

En investigaciones como las de Dvir, Raz y Shenhar (2003) y Faniran, Oluwoye y Lenard (1994), se concluye que reducir la incertidumbre mediante la planeación de los proyectos contribuye a lograr los objetivos, en tanto que el tiempo invertido en estos procesos se recompensa con el éxito de los proyectos.

Por su parte, González, Solís y Alcudia (2010) establecen que la falta de planeación conduce a no contemplar riesgos que finalmente causan incumplimiento en tiempos de entrega. Una de las principales razones que identificó Meister (2006) para no realizar proyectos exitosos es la poca coordinación y comunicación en las fases de iniciación del proyecto entre los gerentes, en tanto que Varajão, Domínguez, Ribeiro y Paiva (2014) encuentran que una correcta definición de los procesos de iniciación y planeación permite alcanzar los objetivos y las metas fijados.

A su vez, Vargas (2015) expone en un artículo la existencia de una brecha importante entre las metodologías, guías, normas y prácticas actuales para la gerencia de proyectos enfocada en la construcción y la ejecución real de los proyectos; por esto, recomienda hacer divulgación e investigación con las entidades privadas, para optimizar el manejo y maximizar el control de proyectos.

Como lo plantean Ahlemann, El Arbi, Kaiser y Heck (2013), no existe la costumbre de utilizar un desarrollo teórico fundamentado en las investigaciones sobre gerencia de proyectos, por lo cual se crea la necesidad de buscar variables principales aplicadas por las pymes dedicadas al sector de la construcción edificatoria, usando como base los dos procesos del grupo de iniciación y los veinticuatro procesos del grupo de la planeación contemplados en el PMBOK 5. ${ }^{\mathrm{a}}$ edición.

Por tal razón, en el presente artículo, que se desarrolla en el marco de la Maestría en Desarrollo y Gerencia Integral de Proyectos de la Escuela Colombiana de Ingeniería Julio Garavito, se pretende hacer el diagnóstico sobre las prácticas en las áreas de iniciación y planeación en gerencia de proyectos para pymes del sector de la construcción en Bogotá.

Igualmente, se busca resolver los problemas existentes mediante la creación de un marco de referencia que permitirá identificar el conjunto de prácticas de gerencia de proyectos aplicables a las pymes del sector de la construcción edificatoria, de tal manera que se puedan hacer lineamientos de gerencia de proyectos que se puedan usar para la creación de un modelo futuro de gerencia de proyectos para pymes. Por lo anterior se formula la siguiente pregunta de investigación: ¿La gerencia de proyectos en pymes

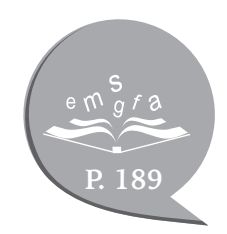


del sector de la construcción edificatoria en Bogotá implementa actualmente prácticas de gerencia de proyectos en sus etapas de iniciación y planeación de proyectos?

El objetivo del presente artículo es hacer un diagnóstico de la aplicación de prácticas en la iniciación y planeación de gerencia de proyectos en pymes del sector de la construcción en Bogotá, con el fin de hallar variables que puedan servir como punto de partida para crear un modelo futuro para la gerencia de proyectos aplicable a pymes. La investigación se empezó con el estudio de los procesos de iniciación y planificación planteados en las principales guías, estándares y prácticas en gerencia de proyectos, como PRINCE2, PMBOK, ICB y P2M. Se destaca la extensión del PMBOK dedicada a la gerencia de proyectos para la construcción en su tercera edición. Posteriormente, se hizo una revisión bibliográfica en artículos indexados y revistas especializadas en el sector, mediante las principales bases de datos bibliográficas, tras lo cual se obtuvo una lista inicial de 181 documentos, de los cuales 80 se volvieron de lectura obligatoria. Esta información se contextualizó con la actualidad colombiana, lo que permitió, en conjunto, realizar el estado del arte. Para ello, se definen los siguientes objetivos específicos:

- Determinar las prácticas aplicadas en los grupos de procesos de iniciación y planeación de gerencia de proyectos en pymes del sector de la construcción inmobiliaria en Bogotá.

- Generar el diagnóstico con respecto a las prácticas descritas en el PMBOK 5. ${ }^{a}$ edición.

- Definir variables que sirvan como punto de partida para la creación de un modelo futuro para la gerencia de proyectos en pymes del sector de la construcción de vivienda residencial y no residencial en Bogotá, tomando como marco los estándares de iniciación y planeación propuestos por el Project Management Institute (PMI) ${ }^{\circledR}$.

\section{Revisión de literatura}

$E_{\mathrm{d} s}^{\mathrm{n}}$ n el desarrollo del marco teórico, se hizo una descripción general de las guías, las normas o los estándares más relevantes en gerencia de proyectos, al tiempo que se efectuó el análisis particular sobre publicaciones académicas. Así mismo, se hizo un acercamiento especial a las investigaciones que se llevaron a cabo para Colombia en los campos de gerencia de proyectos, pymes, y lo referente a los grupos de procesos de iniciación y planeación en gerencia de proyectos.

En atención al avance que ha tenido la gerencia de proyectos en el ámbito mundial y la nueva perspectiva y fortalecimiento que se está dando en el país, se presentan las principales metodologías, normas, guías o estándares para la gerencia de proyectos a escala internacional, que incluyen un poco las metodologías ágiles que se han desarrollado en materia de software y sistemas, en especial.

Entre los estándares más reconocidos en la gerencia de proyectos, se pueden nombrar:

- $\quad$ El PMBOK 5. ${ }^{\mathrm{a}}$ ed. (2013). Guide, PMI (Project Management Institute).

- Extensión para la construcción (2009), $3 .^{\mathrm{a}}$ ed.

- PRINCE2, 2009 edition (2009), OGC (Office of Government Commerce). 
- ICB-IPMA, version 3.0 (2006) (International Project Management Association).

- P2M-PMAJ, vol. I, revisión 3 (2005) (Project Management Association of Japan).

- ISO 21500, versión 2012 (2012), de ISO (International Organization for Standardization).

- SCRUM, versión 2.5 (2014), metodología ágil.

Estos estándares son importantes debido a que recogen un conjunto de buenas prácticas encontradas en la gerencia de proyectos. Morris y Pinto (2004) destacan la importancia del reconocimiento a la gerencia de proyectos como una profesión basada correctamente en guías, normas, buenas prácticas, entrenamiento, conocimiento de los gerentes de proyectos y la estandarización de una terminología común para esta disciplina.

Más allá de las guías formales de gerencia de proyectos, varios investigadores han centrado sus esfuerzos en campos académicos y sectores, donde el papel del gerente de proyectos es fundamental. Las investigaciones relacionadas con pymes abarcan el mayor número de estudios encontrados, debido a que la definición general es ampliamente aceptada en el mundo, con algunos cambios en la estructura referente al número de empleados contratados, los ingresos anuales y la moneda. Las investigaciones suelen ser de carácter descriptivo, las cuales se enfocan en particular en casos de estudio o sectores muy específicos, como alta tecnología (Murphy y Ledwith, 2007), artes gráficas (Rubiano y Cuadros, 2012), industria manufacturera (Tannock, Krasachol y Ruangpermpool, 2002) y construcción (González, Solís y Alcudia, 2010). Cabe señalar que el mayor número de estudios se hacen en el ámbito internacional, donde la investigación se encuentra orientada a casos de estudio de países en particular, como Nigeria (Unit, 2014), Grecia (Sdrolias, Sirakoulis, Trivellas y Poulios, 2005), Escocia (Marcella y Rowley, 2014), España (Aragón y Rubio, 2005), Colombia (Vega, Castaño y Mora, 2011), lo que corrobora la amplia investigación existente al respecto. Dentro del gran número de investigaciones aplicadas a pymes, están Lo y Humphreys (2000), Rahman (2001) y Ghobadian y Gallear (1996 y 1997). Estas investigaciones tienen en común la identificación de elementos claves de éxito para las pymes, y asocian la adopción de prácticas de calidad al éxito de las compañías.

Según Quesado, Aibar y Lima (1989), citados por Andersen, Cobbold y Lawrie (2001), la planificación estratégica puede mejorar el desempeño de las pymes, asegurando el equilibrio entre los objetivos operativos a corto plazo y los objetivos estratégicos a largo plazo, para lo cual se requiere un cuadro de mando integral óptimo y solidificado para la toma de decisiones; sin embargo, lo anterior sí lo cumplen en gran medida las grandes empresas. Además de la planeación, en las investigaciones internacionales, se mencionan estudios referentes a la importancia en la implementación de tecnologías para la gerencia de proyectos, como los de Baumol (2002); Geroski y Machin (1992); Iglesias, Somohano, Rosario, Papis y Rodrigues (2012), y Madrid y García (2008), que sugieren que la innovación produce un aumento de la rentabilidad y un crecimiento de la empresa.

En cuanto al sector de la construcción, se destacan los trabajos realizados por Warszawski (1984), que indagaba la falta de investigación en la construcción y cuestionaba los pocos esfuerzos que se hacían a favor de la investigación, al igual que lo hacen Diez-Silva, Pérez Ezcurdia y Gimena Ramos (2012). En proyectos de construcción, es importante medir el rendimiento, ya que es esencial en el proceso de control, puesto que permite vigilar el desempeño al alcanzar 
objetivos finales (Haponava y Al-Jibouri, 2009). Entre los estudios realizados en los grupos de iniciación y planeación, sobresale el presentado por Platje, Seidel y Wadman (1994), que hacen un análisis de las organizaciones que llevan a cabo múltiples proyectos dentro de la figura de los programas, destacando la importancia de la comunicación entre niveles, desde la iniciación. La comunicación es la base de los proyectos hasta alcanzar el objetivo de planificar el portafolio. Esta investigación ha servido de base a artículos como el de Elonen y Artto (2003), en el que se identifica la inadecuada definición y planeación de los proyectos como una de sus causas; la investigación de Meskendahl (2010), que se adentra en la influencia de la estrategia de negocio de la organización en la planeación de los proyectos; el estudio de Son y Rojas (2010), que analiza el impacto del sesgo de optimismo organizacional en los procesos de planeación de las empresas, así como el artículo de Faniran, Oluwoye y Lenard (1994), que investiga directamente en planeación y construcción; Lehtonen y Martinsuo (2008) investigan la importancia de expandir los límites de la teoría de la organización y hacen hincapié en el valor que le imprime la organización al proyecto durante las primeras etapas de los programas, acentuando la trascendencia del rol de los gerentes de proyecto en esas primeras etapas.

Por último, en la correlación entre gerencia de proyectos, planeación, construcción y pymes, se destacan las investigaciones realizadas por González, Solís y Alcudia (2010), quien presenta un acercamiento al diagnóstico de las prácticas de planeación y control para las pymes en su estudio exploratorio para empresas de la construcción en Yucatán -MéxicoRomero, Melgarejo y Vera-Colina (2015), en su investigación sobre el motivo de fracaso de las pymes para Colombia, señalan que las variables financieras que más impactan el desarrollo de las pymes son la liquidez, la rentabilidad y el nivel de endeudamiento. Por otro lado, Meister (2006) encuentra que una de las principales fallas para alcanzar el éxito en las pymes es la baja definición de los objetivos del proyecto, debido a la poca coordinación y comunicación en las etapas tempranas, en particular en la fase de iniciación, entre los gerentes de proyecto.

\section{Método}

$\mathrm{E}_{\mathrm{e}}^{\mathrm{s}}$ sta sección describe, el diseño metodológico empleado, los participantes o actores involucrados en la investigación, así como el tamaño y tipo de la muestra, los instrumentos y técnicas de investigación aplicadas y los procedimientos empleados desde el punto de vista de las etapas en que se desarrolla la investigación.

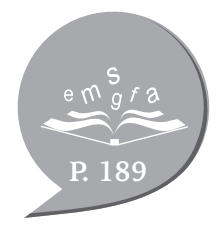

\subsection{Diseño y procedimientos}

La investigación, de tipo cualitativo con alcance exploratorio, se realizó en cinco etapas (figura 1), desde el planteamiento del problema, pasando por la revisión de la bibliografía, trabajo de campo, análisis de datos y, por último, la elaboración del reporte de resultados. 
En las primeras etapas de la investigación, se indagó la necesidad, la oportunidad o el problema por resolver; posteriormente, se desarrolló el árbol de problemas, que arrojó como resultado el planteamiento de la pregunta de investigación, que debe estar alineada con el fortalecimiento de la investigación científica y tecnológica.

Figura 1. Etapas de la investigación

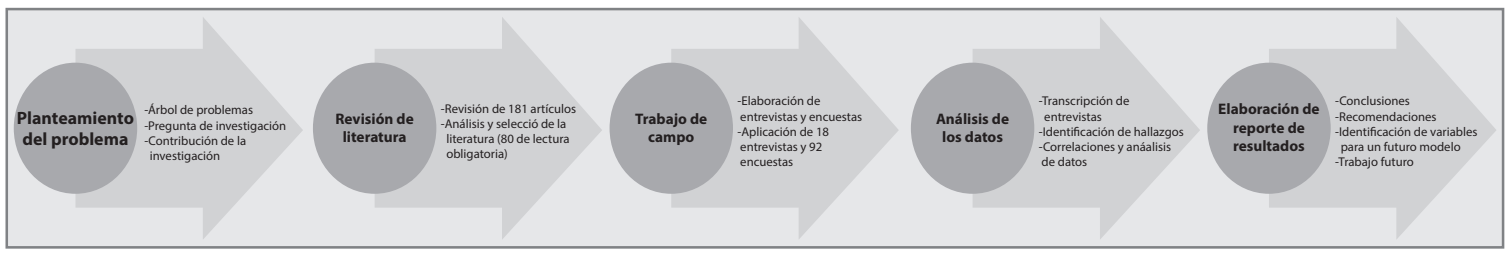

Fuente. Elaboración propia.

Con el fin de hallar artículos relevantes para la investigación, se hizo un estudio preliminar en dos de las bases de datos bibliográficas y de citas de artículos más importantes académicamente: Scopus y Web of Science. Scopus contiene en su registro a varias de las principales editoriales de contenido académico como Elsevier, Springer, Wiley, Taylor \& Francis y Sage.

Además, se buscó información relevante en publicaciones especializadas en la construcción, como Engineering, Construction and Architectural Management e International Journal of Construction Management. Antes de hacer uso de las bases de datos, se establecieron palabras claves para la investigación, como pymes, SME, construcción, iniciación, planeación, Colombia y Bogotá.

Se recolectaron 181 documentos académicos, entre investigaciones académicas, tesis de maestría y tesis de doctorado. Las primeras 80 lecturas académicas entrarían en el grupo inicial de lecturas obligatorias y darían una descripción holística de la actual situación de la planificación de proyectos en las pymes del sector de la construcción en Bogotá, debido a que es el primer paso del proceso de la investigación científica descrito por López (2006).

\subsection{Instrumentos}

En materia de trabajo de campo, se elaboraron entrevistas semiestructuradas a profundidad y encuestas estructuradas enviadas por correo electrónico, aplicadas a gerentes de proyectos o a los que hagan su función en pymes del sector de la construcción edificatoria en Bogotá. En la investigación se adoptó como modelo el par de procesos del grupo de iniciación y los 24 procesos del grupo de planeación contemplados en el PMBOK 5. a ed. En lo relacionado con metodología, se tomó como referencia lo expuesto por Hernández, Fernández y Baptista (2010) para el análisis de datos obtenidos de las entrevistas y la estructuración de las encuestas.

Las entrevistas y encuestas se sometieron a discusión mediante reuniones sostenidas con el grupo de asesores e investigadores, en las que participaron profesores de la Unidad de Proyectos de la Escuela Colombiana de Ingeniería Julio Garavito, profesionales en gerencia de proyectos y los profesores Germán Giraldo -ingeniero industrial, MSc en Ingeniería Industrial, miembro de la American Society for Quality (ASQ), la Society of Hispanic Professional Engineers (SHPE), el Institute of Industrial Engineers (IIE) y el Project Management Professional 63 (PMP) - y César Leal —ingeniero civil, 
especialista en Recursos Hidráulicos y Medio Ambiente, magíster en Ingeniería y Gerencia de Proyectos de Construcción, Project Management Professional (PMP), experto en planeación y control de proyectos-, con el propósito de eliminar información duplicada y obtener respuestas similares por parte de los futuros entrevistados. Posteriormente, los investigadores y el asesor externo de la Unidad de Proyectos de la Escuela Colombiana de Ingeniería, Javier Ramírez Flórez - mercadólogo, especialista en Psicología del Consumidor y asesor en investigación de mercados-, se reunieron para analizar las preguntas planteadas inicialmente.

Por último, con los criterios establecidos por los expertos, se obtuvo un cuestionario para la entrevista de 26 preguntas abiertas principales, de la cuales 11 son secundarias - guías-que sirven de apoyo. La encuesta estructurada tuvo 9 preguntas cerradas con única respuesta y 15 preguntas cerradas de múltiple respuesta, para un total de 24 preguntas. Cada tema de interés se indagó mediante mínimo dos preguntas.

\subsection{Participantes}

Las entrevistas se efectuaron a 18 gerentes de proyectos - $\mathrm{O}$ a quien hace sus labores-, y se obtuvieron 92 respuestas a la encuesta dentro de un conjunto de pymes del sector de la construcción en Bogotá. Una vez realizadas las entrevistas, se comenzó con el trabajo de diagnóstico de la información, tomando como base lo establecido por Hernández, Fernández y Baptista (2010), que definen un hallazgo principal por cada pregunta y unos hallazgos secundarios. Posteriormente, se elaboraron mapas conceptuales para cada una de las preguntas, ya que son herramientas que permiten representar y organizar el conocimiento en forma gráfica (Novak, 1991).

Se identificaron los resultados de las encuestas, plasmados en figuras y datos numéricos. Finalmente, se analizaron los hallazgos y resultados, y se llegó a conclusiones, recomendaciones y variables que servirán como punto de partida para un futuro modelo en la pymes pertenecientes a este importante sector. 


\section{Resultados}

Ta aplicación de los instrumentos a las Lempresas participantes dedicadas a la construcción edificatoria en Bogotá permitió identificar tres estrategias de negocio claramente definidas: una dedicada a proyectos de contratación pública, otra que ejecuta proyectos de contratación netamente con entidades privadas y la última que se especializa en desarrollo y ejecución de proyectos de inversión.

De acuerdo con las encuestas, el 44,6 \% de las empresas son pequeñas y el $25 \%$ son de tamaño mediano; para la presente investigación, se obtuvieron trece entrevistas de organizaciones pequeñas y cinco de compañías medianas. Igualmente, se determina que las organizaciones con más de diez años de experiencia representan un $48,9 \%$, y las que tienen entre uno y cinco años son el 28,3\%. Según entrevistas realizadas, se establece que cinco organizaciones tienen un perfil público, cinco de perfil privado $y$, finalmente, ocho de inversión.
Así mismo, se ha podido determinar que algunas organizaciones implementan parcialmente la guía del PMBOK y que la mediana empresa tiene una mejor cultura en gerencia de proyectos. Además, el 48,9 \% no hacen uso de metodologías o guías para este tipo de gerencia. Tanto la mediana como la pequeña empresa hacen estructuraciones internas, basadas en la experiencia y robustez organizacional. De acuerdo con varias encuestas, en el $70 \%$ de las pymes, se tiene el perfil de gerentes de proyectos; sin embargo, en la mayoría de las organizaciones, esta función la desempeña el propietario de la empresa, gerente general o socio del proyecto.

El 78,3\% de los encuestados manifiestan que actualmente en Colombia no existe cultura en gerencia moderna de proyectos, pero a continuación se muestra que las áreas que tienen mejores prácticas son gestión del tiempo, costo y adquisiciones (figura 2 ). Por otro lado, se pueden apreciar las áreas de poca o nula gestión: identificación, análisis y evaluación de riesgos y gestión de interesados - stakeholders(figura 3).

Figura 2. Buenas prácticas

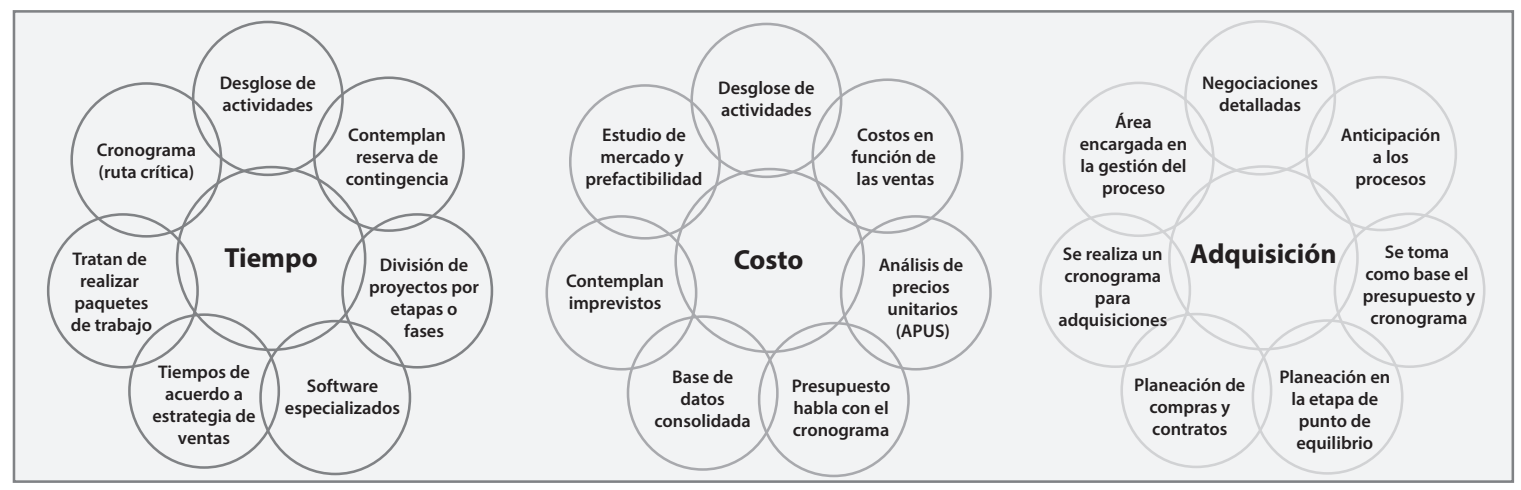

Fuente. Elaboración propia. 
Figura 3. Malas prácticas

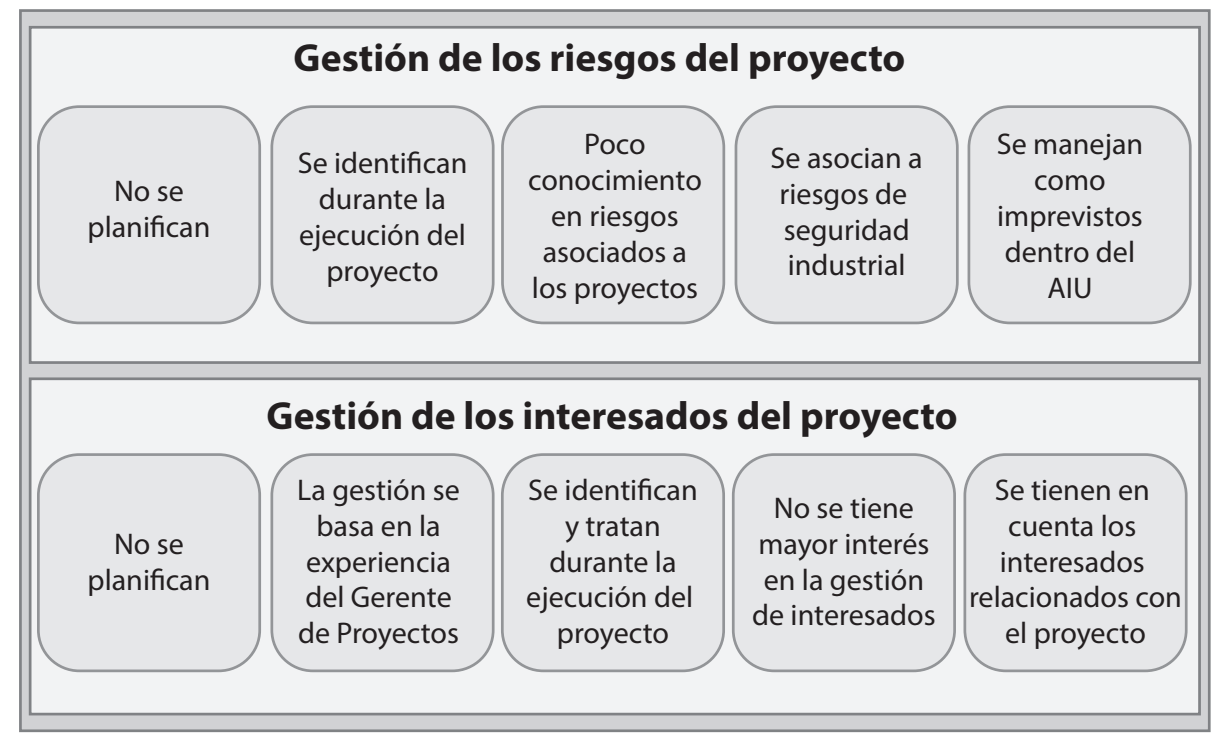

Fuente. Elaboración propia.

El 13,6 y $31,4 \%$ de las pymes no hacen gestión del riesgo y asocian esta área a riesgos de seguridad industrial y salud ocupacional. En cuanto a la identificación y el tratamiento de interesados, el $20 \%$ de las pymes lo realizan en la ejecución del proyecto y el $17 \%$ no hacen ningún tipo de planificación.

\subsection{Organizaciones con estrategia de negocio para proyectos de contratación pública}

Se caracterizan por tratarse de licitaciones públicas y otros procesos de selección, en las que las empresas constructoras compiten por la adjudicación de un contrato de construcción, dependiendo de las políticas de la entidad estatal. Las organizaciones realizan análisis en los ámbitos económico, jurídico, financiero y de experiencia, y una vez entregadas las propuestas por parte de los oferentes, la entidad pública empieza el proceso de evaluación y adjudicación del proyecto. Luego de su adjudicación, la pyme comienza con una planeación básica, la cual se ve limitada por el corto plazo para su desarrollo. Para ello, se comienza con la constitución del acta de inicio y con un análisis en materia de tiempo y costo, definido por la entidad pública. Cabe señalar que la mayoría de los procesos de planeación se llevan a cabo en la etapa de ejecución del proyecto.

Entre los hallazgos de prácticas ejercidas por las organizaciones, se encuentran buenas prácticas como la gestión del tiempo, el costo, las adquisiciones y las compras, en tanto que prácticas como calidad, recurso humano y comunicaciones deben reforzarse y mejorarse, a diferencia de la gestión de riesgos e interesados, que tienen poca o nula gestión. 


\subsubsection{Procesos de gestión}

En lo relacionado con tiempo y costo, se pudo detectar que hacen una adecuada gestión de los procesos mediante desglose de actividades, pero no realizan identificación, análisis y evaluación de riesgos, lo que repercute en permanentes desviaciones durante la ejecución del proyecto. Las organizaciones manifiestan que la gestión de riesgos no está contemplada dentro de los alcances contractuales del contrato y además recomiendan que las entidades estatales implementen métodos o guías para reducir las desviaciones e incertidumbres en la etapa de planeación.

Para cubrir imprevistos, las organizaciones definen un porcentaje del costo directo para cubrir contingencias, imprevistos y riesgos, en general por medio de administración, imprevistos y utilidad (AIU) del contrato; así, la planificación del costo se reduce a cumplir con el presupuesto establecido por el cliente. Por otra parte, la constante fluctuación de precios de materiales y mano de obra repercute en la variabilidad del costo, lo que implica desviaciones en los costos finales del proyecto, los cuales se identifican durante la ejecución del proyecto y no durante su planificación; esto impide tomar medidas o controles. Igualmente, la falta de cumplimiento en el pago de las entidades públicas repercute en el flujo de caja de los proyectos.

Un flujo de caja estable y sólido para este tipo de organizaciones es fundamental, razón por la cual uno de los objetivos es minimizar las desviaciones en costos; las empresas recomiendan elaborar un buen análisis del presupuesto inicial mediante la creación de un área encargada para tal fin, en la que se tengan en cuenta lecciones aprendidas (Project Management Institute [PMI], 2013), y como buenas prácticas aconsejan analizar previamente variables económicas como índice de precios al consumidor (IPC), inflación, dólar y precio del petróleo.
Las organizaciones hacen una adecuada planeación en el proceso de compras y contratos. Aunque no manejan una guía o estándar, en la empresa tratan de estructurar la gestión de proyectos basados en la anticipación para la ejecución de actividades y la programación del proyecto. Riesgos en el ámbito económico, inestabilidad jurídica y gubernamental, corrupción, entre otros, son factores que afectan la buena gestión de adquisiciones dentro de las pymes.

\subsubsection{Procesos por mejorar}

En cuanto a calidad, se identificó que en la mayoría de las organizaciones no se realiza planeación de la gestión de la calidad y esta se elabora durante la ejecución del proyecto. En ciertas ocasiones, las entidades públicas exigen un plan de gestión de calidad, pero en otras oportunidades se visualiza como un gasto y no como una inversión (Ghobadian y Gallear, 1996), ya que en la mayoría de los casos no se cuenta con un área de gestión y por lo general el ingeniero encargado de la ejecución del proyecto hace la gestión de calidad.

La gestión de recursos humanos tiene, en gran medida, parámetros similares a los establecidos en el PMBOK 5. ${ }^{a}$ ed. Se destaca que la construcción, al ser una industria dinámica, repercute en la constante rotación del personal; por tal motivo, el personal requerido para la ejecución del proyecto no es directo de planta, sino personal externo con la experiencia suficiente, dependiendo de las especificaciones y el alcance del producto.

Se identificó que dentro de las organizaciones no se planean las comunicaciones y que hacen la gestión durante la ejecución del proyecto, pero sí utilizan estrategias de divulgación y herramientas de comunicación, como las descritas en el PMI (2013). La falta de una comunicación fluida, veraz y oportuna es quizá uno de los grandes problemas detectados; por eso, se requieren canales de comunicación más 
fluidos, como informes de desempeño claros y eficaces, al igual que estrategias para mejorar la comunicación interpersonal, que pueden ayudar a fortalecer el proceso.

\subsubsection{Poca o nula gestión}

La planeación y gestión de riesgos es el proceso que tiene menor desarrollo, aparte del hecho de que hay falta de planeación en los riesgos (Verbano y Venturini, 2013). Dentro del marco de la contratación estatal, el ente contratante es quizá el que debe proveer esta planeación, pero ya sea por falta de cultura, de recursos económicos o por desconocimiento, este proceso no tiene la trascendencia necesaria en la gerencia de proyectos. Con el fin de mejorar esta práctica, las empresas coinciden en que los entes estatales deberían desarrollar una adecuada planeación en la gestión de riesgos o incluir este proceso en los requerimientos de contratación, donde se estipule un tiempo y costo para su desarrollo.

El tratamiento de los riesgos que en general utilizan las organizaciones que contratan con el Estado consiste en transferir el riesgo al ente contratante, mediante el pago de una mayor cantidad de obra, la prórroga de tiempo, el pago de actividades no contempladas o modificaciones que se presenten en el alcance. A continuación, se identifican las variables claves en los procesos del PMBOK 5. ${ }^{a}$ ed. (tabla 1).

Tabla 1. Variables del perfil público

\begin{tabular}{|c|c|c|}
\hline Perfil & $\begin{array}{c}\text { Área de } \\
\text { conocimiento }\end{array}$ & Variable \\
\hline \multirow{4}{*}{ Público } & Integración & $\begin{array}{l}\text { - Tener contratos y órdenes de servicio, anexando documentos claros que definan el alcance } \\
\text { y las restricciones del caso. } \\
\text { - Estipular los tiempos en fases de planeación y ejecución del proyecto. } \\
\text { - Definir cómo se controlarán las fases. } \\
\text { - Establecer cómo será el cierre del proyecto. } \\
\text { - Realizar reunión kick-off, documentada mediante acta, en la que se definan tareas y } \\
\text { responsables. }\end{array}$ \\
\hline & Alcance & $\begin{array}{l}\text { - Solicitar información técnica, económica y social del proyecto. Si no los hay, debe quedar } \\
\text { registrado. } \\
\text { - } \text { Registrar deficiencias, fortalezas y restricciones. }\end{array}$ \\
\hline & Tiempo & $\begin{array}{l}\text { - } \text { Definir tiempos para cada actividad. } \\
\text { - Registrar si la entidad contratante contempló riesgos en el cronograma y si forma parte } \\
\text { del contrato. } \\
\text { - Establecer reservas de tiempo en el cronograma. } \\
\text { - Planificar y desarrollar flujo de caja en materia de tiempo. }\end{array}$ \\
\hline & Costos & $\begin{array}{l}\text { - Hacer cotejo de actividades con el cronograma fijado. } \\
\text { - Registrar si la entidad contratante contempló riesgos en cuanto a costos y si forma parte } \\
\text { del contrato. } \\
\text { - Establecer reservas en costos y dejar registro del contrato. } \\
\text { - Planificar y desarrollar flujo de caja en materia de costo. }\end{array}$ \\
\hline
\end{tabular}


Tabla 1. Variables del perfil público (Continuación)

\begin{tabular}{|c|c|c|}
\hline Perfil & $\begin{array}{c}\text { Área de } \\
\text { conocimiento }\end{array}$ & Variable \\
\hline \multirow{7}{*}{ Público } & Calidad & $\begin{array}{l}\text { - Planificar la gestión de la calidad mediante un enfoque en calidad de recursos humanos y } \\
\text { contrataciones. } \\
\text { - Garantizar el cumplimiento con las normas reguladoras en la construcción de edificaciones. } \\
\text { - Definir seguimientos a las actividades mediante auditorías internas del personal más ex- } \\
\text { perimentado. } \\
\text { - Determinar, desde el nacimiento del proyecto, los controles claves de calidad mediante una } \\
\text { lista de chequeo. } \\
\text { - Hacer, en la medida de lo posible, procesos de certificación con entidades externas o, en } \\
\text { su defecto, establecer una estructura interna de calidad por medio de auditorías y visitas } \\
\text { de obra. }\end{array}$ \\
\hline & Recurso humano & $\begin{array}{l}\text { - Definir los perfiles de cargo para la ejecución del proyecto. } \\
\text { - Establecer la necesidad de recurso humano basado en la planeación del proyecto. } \\
\text { - Contar con bases de datos de personal especializado en el sector. } \\
\text { - Diseñar estrategias que ayuden a minimizar la rotación del personal. } \\
\text { - Diseñar estrategias para mejorar los ambientes laborales. }\end{array}$ \\
\hline & Comunicaciones & $\begin{array}{l}\text { - Definir un plan de comunicación desde el nacimiento del proyecto. } \\
\text { - Establecer los perfiles que generen liderazgo y contemplar estrategias de comunicación } \\
\text { efectiva. } \\
\text { - Informar a todo el equipo de trabajo objetivos, alcances, beneficios y avances del proyecto. }\end{array}$ \\
\hline & Riesgos & $\begin{array}{l}\text { - Identificar, planificar, evaluar y registrar los riesgos, y divulgarlos a todo el equipo de tra- } \\
\text { bajo. } \\
\text { - Tener presente que la gestión de riesgos debe hacerla el personal que cuente con el know- } \\
\text { how de la organización. } \\
\text { - Elaborar un registro de riesgos. }\end{array}$ \\
\hline & Adquisiciones & $\begin{array}{l}\text { - Establecer contratos y servicios en los mismos términos que la organización los haya fir- } \\
\text { mado con el contratante. } \\
\text { - Contar con una base de datos clave de proveedores. } \\
\text { - Programar compras y contratos, según los tiempos del cronograma del proyecto. } \\
\text { - Planificar el flujo de caja para las adquisiciones y garantizar que este se materialice. } \\
\text { - Identificar riesgos por la dinámica económica del país. } \\
\text { - Tener en cuenta que las adquisiciones debe manejarlas personal de absoluta confianza. }\end{array}$ \\
\hline & Interesados & $\begin{array}{l}\text { - Definir con la entidad contratante quién es el encargado de la identificación, la caracteri- } \\
\text { zación y el relacionamiento con los stakeholders. } \\
\text { - Dejar registro de quién asume los contratiempos que se puedan presentar por la gestión } \\
\text { de interesados. }\end{array}$ \\
\hline & $\begin{array}{l}\text { Seguimiento y } \\
\text { control }\end{array}$ & $\begin{array}{l}\text { - Definir procedimientos claros en controles de cambio. } \\
\text { - Solicitar doble verificación a controles de cambio, con registro de quién pide el cambio y } \\
\text { quién lo autoriza. } \\
\text { - Garantizar la transferencia de conocimiento entre proyectos o tipos de negocio mediante } \\
\text { un procedimiento de lecciones aprendidas. }\end{array}$ \\
\hline
\end{tabular}

Fuente. Elaboración propia. 
De acuerdo con lo manifestado por los entrevistados, se recomienda identificar y planificar las relaciones con los interesados para mitigar posibles stakeholders que afecten el proyecto.

Fuera de los procesos de planeación, se indagaron prácticas ejercidas en el grupo de monitoreo y control, donde se identificaron gestión basada en actas de obra, anotaciones al libro de obra y divulgación por correo electrónico. Con respecto a las medidas que se implementan en desviaciones, se hacen acciones correctivas y se llevan a cabo planes de contingencia de acción inmediata. No se identificó una planificación en las posibles desviaciones que el proyecto pueda presentar, y como actualmente no se acostumbra hacer este análisis, no se tiene un registro de lecciones aprendidas estructurado.

\subsection{Organizaciones con estrategia de negocio para proyectos de contratación privada}

Se caracterizan por ejecutar proyectos de índole privada, en los que las empresas ejecutoras se basan en las definiciones de alcance, tiempo y costos emitidas por el cliente. Posteriormente, se analiza y valida el cumplimiento de los requerimientos y las necesidades del contratante, y se procede a realizar el contrato, en el que se plasman los alcances finales del proyecto que se va a ejecutar.

Al igual que la estrategia de negocio en el ámbito público, se detectaron procesos de buena gestión, otros que requieren mejora y algunos cuya gestión es nula. Entre los problemas generales, están una incorrecta definición del alcance y las limitaciones económicas por parte del cliente. Como recomendación general, se contempla tener solidez económica, técnica y jurídica en los procesos de contratación donde se evidencie una definición clara del alcance del proyecto.

\subsubsection{Procesos de gestión}

La optimización en la gestión de tiempo se contempla como factor de éxito, motivo por el cual la estimación es más estricta y limitada. Se establece un correcto desglose de actividades, por lo general alineado a los costos. Como problemas se pudieron detectar alcances deficientes, falta de identificación de riesgos que perjudican los tiempos para la ejecución del proyecto, cronogramas no detallados y seguimiento inapropiado en el cronograma.

La gestión de costos es óptima, ya que se hace un análisis de precio unitario (APU) a cada actividad; no es recomendable contemplar un porcentaje de imprevistos dentro del concepto AIU sin realizar identificación y evaluación de riesgos. Se recomienda que las pymes tengan un área exclusiva de costos, tratar en lo posible de generar un presupuesto y programación con los mismos parámetros de desglose paquetes de trabajo-, al igual que invertir en software para la planeación y el control presupuestal. Por último, tener información veraz y oportuna en la toma de decisiones para la estimación de variables económicas.

La gestión de adquisiciones se realiza en una forma ordenada, ya que su enfoque es la optimización en las negociaciones. Se recomienda realizar programación independiente de compras y contratos, así como tener personal encargado con experiencia técnica $\mathrm{y}$ administrativa.

\subsubsection{Procesos por mejorar}

Limitaciones de tiempo e interventorías poco participativas son quizá los problemas generales en la gestión de la calidad. Por otra 
parte, al tener un enfoque de negocio privado, es fundamental crear gestión de calidad que genere poco esfuerzo operativo y optimice la ejecución del proyecto.

Las organizaciones privadas hacen la gestión de recursos humanos basada en el tipo de construcción, magnitud y alcance del proyecto. El gran reto de las organizaciones es establecer programas de incentivos, actualización académica y motivación, con el fin de minimizar la rotación de personal y fortalecer el compromiso del personal.

Las pymes identificaron la necesidad de hacer una planeación o una estrategia de comunicaciones, pero actualmente esta gestión no se lleva a cabo. El proceso se desarrolla en la ejecución y se basa, por lo general, en la experiencia de los encargados.

\subsubsection{Poca o nula gestión}

En cuanto a la gestión de riesgos, la diferencia con la pública es que las organizaciones de carácter privado tienen un conocimiento básico de este proceso, pero las empresas no la desarrollan por planeaciones aceleradas, limitación presupuestal o desconocimiento.

Entre los aspectos generales, cabe mencionar que los interesados dependen de la magnitud del proyecto, que las estrategias de manejo se plantean en la ejecución y que se trata de hacer una gestión de comunicación y relacionamiento básica con los interesados en el proyecto (Tabla 2 ).

Tabla 2. Variables de perfil privado

\begin{tabular}{|c|c|c|}
\hline Perfil & $\begin{array}{c}\text { Área de } \\
\text { conocimiento }\end{array}$ & Variable \\
\hline \multirow{4}{*}{ Privado } & Integración & $\begin{array}{l}\text { - Oficializar el proyecto desde su nacimiento mediante un documento formal. } \\
\text { - Estipular los tiempos en fases de planeación y ejecución del proyecto. } \\
\text { - Definir cómo se controlarán las fases. } \\
\text { - Definir cómo será el cierre del proyecto. } \\
\text { - Realizar reunión kickoff, documentada mediante acta, para definir tareas y responsables. }\end{array}$ \\
\hline & Alcance & $\begin{array}{l}\text { - Celebrar una reunión para aclarar el alcance y las restricciones del proyecto, y dejar docu- } \\
\text { mentados los cambios o las adiciones hechos. } \\
\text { - Establecer el alcance tomando como base el desglose de actividades. }\end{array}$ \\
\hline & Tiempo & $\begin{array}{l}\text { - Definir tiempos en fases del proyecto. } \\
\text { - Revisar los alcances del proyecto y asignar tiempos a cada actividad. } \\
\text { - Identificar los riesgos y los tiempos en caso de materializarse. } \\
\text { - Establecer reservas de tiempo. }\end{array}$ \\
\hline & Costos & $\begin{array}{l}\text { - Revisar el alcance en conjunto con los tiempos fijados. } \\
\text { - Hacer un análisis de los costos. } \\
\text { - Identificar los riesgos y los costos en caso de materializarse. } \\
\text { - Establecer costos por desglose de actividades y los análisis de precios unitarios (APU). }\end{array}$ \\
\hline
\end{tabular}


Tabla 2. Variables de perfil privado (Continuación)

\begin{tabular}{|c|c|c|}
\hline Perfil & $\begin{array}{c}\text { Área de } \\
\text { conocimiento }\end{array}$ & Variable \\
\hline \multirow{7}{*}{ Privado } & Calidad & $\begin{array}{l}\text { - Planificar la gestión de la calidad mediante un enfoque en calidad de recursos humanos } \\
\text { - Garantizar el cumplimiento con las normas reguladoras en la construcción de edificaciones. } \\
\text { - Definir seguimientos a las actividades mediante auditorías internas del personal más } \\
\text { experimentado. } \\
\text { - Establecer desde el nacimiento del proyecto los controles claves de calidad mediante una } \\
\text { lista de chequeo. } \\
\text { - Hacer, en la medida de lo posible, procesos de certificación con entidades externas o, en } \\
\text { su defecto, establecer una estructura interna de calidad por medio de auditorías y visitas } \\
\text { de obra. }\end{array}$ \\
\hline & Recurso Humano & $\begin{array}{l}\text { - Hacer la gestión del recurso humano desde el nacimiento del proyecto. } \\
\text { - Definir los perfiles de cargo para cada fase del proyecto. } \\
\text { - Establecer la necesidad de recurso humano basado en las fases del proyecto. } \\
\text { - Contar con bases de datos de personal especializado en el sector. } \\
\text { - Diseñar estrategias que ayuden a minimizar la rotación del personal. } \\
\text { - Planear estrategias para mejorar los ambientes laborales. }\end{array}$ \\
\hline & Comunicaciones & $\begin{array}{l}\text { - Definir un plan de comunicación desde el nacimiento del proyecto. } \\
\text { - Establecer los perfiles que generen liderazgo y contemplar estrategias de comunicación } \\
\text { efectiva. } \\
\text { - Informar a todo el equipo de trabajo objetivos, alcances, beneficios y avances del proyecto. }\end{array}$ \\
\hline & Riesgos & $\begin{array}{l}\text { - Identificar, planificar, evaluar y registrar los riesgos, y divulgarlos a todo el equipo de } \\
\text { trabajo. } \\
\text { - Procurar que la gestión de riesgos la haga el personal que tenga el know-how de la } \\
\text { organización. } \\
\text { - Elaborar un registro de riesgos. }\end{array}$ \\
\hline & Adquisiciones & $\begin{array}{l}\text { - Establecer contratos y servicios en los mismos términos en que la organización los haya } \\
\text { firmado con el contratante. } \\
\text { - Contar con una base de datos claves de proveedores. } \\
\text { - Programar compras y contratos según los tiempos del cronograma del proyecto. } \\
\text { - Planificar el flujo de caja para las adquisiciones y garantizar que este se materialice. } \\
\text { - Identificar riesgos por la dinámica económica del país. } \\
\text { - Garantizar que las adquisiciones debe hacerlas personal de absoluta confianza. }\end{array}$ \\
\hline & Interesados & $\begin{array}{l}\text { - Identificar los interesados claves para el correcto desarrollo del proyecto. } \\
\text { - Establecer estrategias de relacionamiento con los interesados. } \\
\text { - Procurar que la identificación de interesados y su relacionamiento sea realista. }\end{array}$ \\
\hline & $\begin{array}{l}\text { Seguimiento y } \\
\text { control }\end{array}$ & $\begin{array}{l}\text { - Definir procedimientos claros en controles de cambio. } \\
\text { - Solicitar doble verificación a controles de cambio, con registro de quien solicita el cambio } \\
\text { y quien lo autoriza. } \\
\text { - Concertar los cambios con el cliente. } \\
\text { - Informar al cliente, desde el inicio del proyecto, sobre posibles desviaciones en costos y } \\
\text { ampliación de plazos por cambios que se soliciten. } \\
\text { - Garantizar la transferencia de conocimiento entre proyectos o tipos de negocio mediante } \\
\text { un procedimiento de lecciones aprendidas. }\end{array}$ \\
\hline
\end{tabular}

Fuente. Elaboración propia. 
Las pymes requiere iniciar una cultura de trazabilidad y documentación de los cambios, dado que implementar lecciones aprendidas ayuda en la planeación y el tratamiento de posibles desviaciones o cambios. En lo referente al método de valor ganado, las empresas manifiestan que tienen conocimiento de la metodología, guías o prácticas, pero por factores económicos o por desconocimiento del personal encargado no se aplica.

\subsection{Organizaciones con estrategia de negocio para proyectos de inversión}

Se caracterizan por el desarrollo, la venta/ arriendo y la ejecución de proyectos, apalancados con recursos propios, capital de socios, fondos de inversión, sistema de títulos, entre otros. Por lo general, el proyecto contempla una etapa de estudio de potencialidad, evaluación, preventa - punto de equilibrio- $y$, finalmente, la ejecución del proyecto. Otras organizaciones se enfocan en el arrendamiento y la operación del inmueble.

Las organizaciones que suelen tener este perfil comienzan con la identificación de la oportunidad de negocio o necesidad por satisfacer. Se requiere tener alianzas estratégicas con el sistema bancario para la financiación del proyecto. Una decisión basada en métodos de sensibilidad como debilidades, oportunidades, fortalezas y amenazas (DOFA) o las estrategias de Porter (1980) ayudarán a estructurar mejor el proyecto.

Para la definición del alcance del producto que se va a vender o arrendar, las organizaciones recomiendan analizar detalladamente aspectos gubernamentales, normativos, jurídicos, económicos y de mercado aplicados a proyectos de edificación. El mercado actual presenta poca oferta de lotes y plusvalías altas, lo que limita alcances en el ámbito presupuestal y repercute en modificaciones al producto y, en ciertos casos, en la cancelación del proyecto.

\subsubsection{Procesos de gestión}

La estimación de tiempo se hace desde la idea del producto, prefactibilidad, ventas y ejecución de proyecto, lo que genera una adecuada planeación; además, disponer de un equipo de trabajo capacitado técnica y administrativamente ayuda en la optimización del proceso. Por otra parte, las organizaciones recomiendan desarrollar una programación que hable con el presupuesto basado en la misma estructura de desglose, ya que esto es fundamental para el cumplimiento de índices de desempeño en alcance, tiempo y costo.

La gestión de costos se basa en juicio de expertos, análisis unitarios de cada una de las actividades, análisis de índices y comportamiento del mercado. Se efectúa poco o nulo análisis de riesgos económicos, lo que arroja desviaciones muy grandes en ciertas ocasiones, al tiempo que se destaca que poseer información veraz y oportuna es clave en la evaluación de variables económicas y en el comportamiento del mercado. Hacer índices basados en el método de valor ganado es una buena práctica para ser anticipadores y no improvisadores. El manejo de índices de construcción y estudios de sensibilidad en materia de costo directo e indirecto permite analizar un panorama general del proyecto.

En cuanto al perfil de inversión, hay que hacer una buena planeación de adquisiciones, agilizando el proceso de ejecución del proyecto y contribuyendo con factores de éxito y buenas prácticas. Los problemas en el cumplimiento de subcontratistas, proveedores y limitación de recursos económicos se pueden subsanar con una amplia base de datos y un buen conocimiento de la industria. 


\subsubsection{Procesos por mejorar}

La gestión de calidad está mejor estructurada en comparación con la pública y la privada. Tener certificaciones como la ISO 9001 y la ISO 14000 ayuda en la optimización del proceso. Los proyectos deben cumplir con la normativa vigente (NSR 2010) durante la ejecución. No obstante, las organizaciones pequeñas no tienen solidez económica, lo que lleva a que sean «toderas», es decir, a que hagan sus procesos limitando el personal, el cual debe asumir labores ajenas a su conocimiento y preparación. Se recomienda realizar estructuras internas en la gestión de calidad basadas en los estándares o las guías que se encuentran hoy en día en el mercado.

La gestión de recursos humanos en algunos casos se ve limitada a factores monetarios y baja experiencia en el reclutamiento del personal. Se evidencian falencias en la estructuración y planeación del proceso y, a la vez, se detectan problemas como mercado laboral aleatorio y preparación integral del profesional por parte de la academia.

Pocas organizaciones planean en forma detallada las comunicaciones, ya que su función se fundamenta en dar a conocer las herramientas de divulgación y en tener soporte tecnológico. En cuanto a la comunicación con agentes externos, las organizaciones manifiestan que algunas entidades gubernamentales no son conscientes de los beneficios del desarrollo urbano y social, velando más por el bien propio que por el bien común, lo que despierta insensibilidad hacia la industria.

\subsubsection{Poca o nula gestión}

Los entrevistados manifiestan que a las empresas no les interesa realizar una gestión en este proceso, pese a que son conscientes de la volatilidad económica y el dinamismo de la industria. Para subsanar las posibles desviaciones, contemplan reservas presupuestales y, gracias a la experiencia, implementan planes de contingencia o acciones correctivas. Los riesgos ambientales, jurídicos, normativos y económicos son los más frecuentes.

Las organizaciones manifiestan que los interesados son un punto crítico y fundamental para el proyecto, pero su práctica está basada en la experiencia. Como menciona Meister (2006), una de las principales fallas para alcanzar el éxito es la baja comunicación entre las etapas. Falta de interés y poco conocimiento en la gestión de interesados repercuten en estrategias de divulgación y comunicación con los agentes internos y externos del proyecto.

Con respecto al grupo de monitoreo y control, las organizaciones manifiestan que es esencial en el buen desempeño de los proyectos. Los expertos señalan que la mayoría de las organizaciones contemplan dentro de sus procesos control de alcance, tiempo, costo y calidad, gestión de informes de desempeño, utilización de software en planeación y control, identificación y evaluación de cambios.

Apenas dos de los entrevistados aseguraron que la gestión de control del proyecto la realizan por medio del método de valor ganado. Muchas de las organizaciones son conocedoras del método, pero aducen que, además de no ser muy claro, es complicado de aplicar. Por otra parte, uno de los entrevistados manifestó que el método del valor ganado debe hacerse en función de las ventas y no de la ejecución. Se destaca que la gestión depende del nivel de la empresa, pues las organizaciones medianas tienen procesos mejor estructurados que los de las pequeñas.

A renglón seguido, se muestran los resultados en proyectos de inversión. Vale la pena señalar que estas variables o resultados aplicarían para las pymes que están en el sector de la construcción edificatoria y cumplan con alguna de las estrategias de negocio encontradas en la investigación (Tabla 3). 
Tabla 3. Variables del perfil de inversión

\begin{tabular}{|c|c|c|}
\hline Perfil & $\begin{array}{c}\text { Área de } \\
\text { conocimiento }\end{array}$ & Variable \\
\hline \multirow{7}{*}{ Inversión } & Integración & $\begin{array}{l}\text { - Oficializar el proyecto desde su nacimiento mediante un documento formal. } \\
\text { - Estipular los tiempos en fases de planeación y ejecución del proyecto. } \\
\text { - Definir cómo se controlarán las fases. } \\
\text { - Definir cómo será el cierre del proyecto. } \\
\text { - Realizar reunión kickoff, documentada mediante acta, y definir tareas y responsables. }\end{array}$ \\
\hline & Alcance & $\begin{array}{l}\text { - Utilizar una guía estandarizada para definir el alcance del proyecto - dependiendo de } \\
\text { cuál se va a realizar-, que contenga una lista de chequeo de los criterios del alcance. } \\
\text { - Establecer el alcance del proyecto, tomando como base el desglose de actividades. }\end{array}$ \\
\hline & Tiempo & $\begin{array}{l}\text { - Definir tiempos en fases del proyecto. } \\
\text { - Revisar los alcances del proyecto y asignar tiempos a cada actividad. } \\
\text { - Identificar los riesgos y los tiempos en caso de materializarse. } \\
\text { - Determinar reservas de tiempo. }\end{array}$ \\
\hline & Costos & $\begin{array}{l}\text { - Revisar el alcance en conjunto con los tiempos fijados. } \\
\text { - Hacer un análisis de los costos. } \\
\text { - Identificar los riesgos y los costos en caso de materializarse. } \\
\text { - Establecer costos por desglose de actividades y los APU. }\end{array}$ \\
\hline & Calidad & $\begin{array}{l}\text { - Planificar la gestión de la calidad mediante un enfoque en calidad de recursos hu- } \\
\text { manos y contrataciones. } \\
\text { - Garantizar el cumplimiento con las normas reguladoras en la construcción de edifi- } \\
\text { caciones. } \\
\text { - Definir seguimientos a las actividades mediante auditorías internas del personal más } \\
\text { experimentado. } \\
\text { - Establecer, desde el nacimiento del proyecto, los controles claves de calidad mediante } \\
\text { una lista de chequeo. } \\
\text { - Realizar, en la medida de lo posible, procesos de certificación con entidades externas } \\
\text { o, en su defecto, establecer una estructura interna de calidad mediante auditorías y } \\
\text { visitas de obra. }\end{array}$ \\
\hline & Recurso humano & $\begin{array}{l}\text { - Hacer la gestión del recurso humano desde el nacimiento del proyecto. } \\
\text { - Definir los perfiles de cargo para cada fase del proyecto. } \\
\text { - Establecer la necesidad de recurso humano según las fases del proyecto. } \\
\text { - Contar con bases de datos de personal especializado en el sector. } \\
\text { - Diseñar estrategias que ayuden a minimizar la rotación del personal. } \\
\text { - Diseñar estrategias para mejorar los ambientes laborales. }\end{array}$ \\
\hline & Comunicaciones & $\begin{array}{l}\text { - Definir un plan de comunicación desde el nacimiento del proyecto. } \\
\text { cación efectiva. } \\
\text { - Informar a todo el equipo de trabajo los objetivos, alcances, beneficios y avances del } \\
\text { proyecto. }\end{array}$ \\
\hline
\end{tabular}


Tabla 3. Variables del perfil de inversión (Continuación)

\begin{tabular}{|c|c|c|}
\hline Perfil & $\begin{array}{c}\text { Área de } \\
\text { conocimiento }\end{array}$ & Variable \\
\hline \multirow{4}{*}{ Inversión } & Riesgos & $\begin{array}{l}\text { - Identificar, planificar, evaluar y registrar los riesgos, y divulgarlos a todo el equipo de } \\
\text { trabajo. } \\
\text { - Procurar que la gestión de riesgos la haga el personal que tenga el know-how de la } \\
\text { organización. } \\
\text { - Elaborar un registro de riesgos. }\end{array}$ \\
\hline & Adquisiciones & $\begin{array}{l}\text { - Establecer contratos y servicios en los mismos términos en que la organización los } \\
\text { haya firmado con el contratante. } \\
\text { - Disponer de una base de datos clave de proveedores. } \\
\text { - Programar compras y contratos según los tiempos del cronograma del proyecto. } \\
\text { - Planificar el flujo de caja para las adquisiciones y garantizar que este se materialice. } \\
\text { - Identificar riesgos por la dinámica económica del país. } \\
\text { - Procurar que las adquisiciones las maneje personal de absoluta confianza. } \\
\text { - Planificar las adquisiciones en la fase de punto de equilibrio. } \\
\text { - Centralizar todas las adquisiciones. }\end{array}$ \\
\hline & Interesados & $\begin{array}{l}\text { - Identificar a los interesados claves para el correcto desarrollo del proyecto. } \\
\text { - Establecer estrategias de relacionamiento con los interesados. } \\
\text { - Recordar que la identificación de interesados y su relacionamiento debe ser realista. }\end{array}$ \\
\hline & $\begin{array}{l}\text { Seguimiento y } \\
\text { Control }\end{array}$ & $\begin{array}{l}\text { - Definir procedimientos claros en controles de cambio. } \\
\text { - Solicitar doble verificación a controles de cambio, con registro de quien solicita el } \\
\text { cambio y quien lo autoriza. } \\
\text { - Concertar los cambios con el cliente. } \\
\text { - Informar al cliente, desde el inicio del proyecto, sobre posibles desviaciones en costos } \\
\text { y ampliación de plazos por cambios que se soliciten. } \\
\text { - Garantizar, mediante un procedimiento de lecciones aprendidas, la transferencia de } \\
\text { conocimiento entre proyectos o tipos de negocio. }\end{array}$ \\
\hline
\end{tabular}

Fuente. Elaboración propia.

Los entrevistados manifiestan algunas claves de éxito, como cumplir el alcance, garantizar la calidad del producto entregado, mantener una buena figura con el cliente, realizar una adecuada planeación y anticipación a los procesos, hacer permanente seguimiento y control al proyecto y, por último, tener un conocimiento adecuado del mercado. Entre los resultados obtenidos, las pymes enfocan sus fuerzas en la planeación del tiempo con un $87,70 \%$, luego en el costo con un $85,2 \%$ y finalmente en el alcance con un $46,9 \%$.
Entre las recomendaciones que emiten las pymes, están la evolución del aprendizaje, realizar una gerencia integral de proyectos, optimizar los procesos de planificación, apoyo y capacitación por parte del Estado en gerencia moderna de proyectos, seguimiento y control continuo, conocimiento y manejo del mercado, información veraz, oportuna y de calidad. Finalmente, las empresas toman como criterio de éxito de proyecto el cumplimiento del costo en un $91,90 \%$, seguido del tiempo con un $46,90 \% \mathrm{y}$, por último, el alcance con un $39,50 \%$. 


\section{Discusión}

$\mathrm{D}^{\mathrm{s}}$ e acuerdo con las variables propuestas, las pymes del sector de la construcción en Bogotá podrían desarrollar procesos de gerencia de proyectos para fortalecer los resultados actuales, basados en consideraciones propias del sector y de la práctica de la gerencia moderna de proyectos. El 78,3\% de los participantes consideran que actualmente en Colombia no existe cultura en gerencia moderna de proyectos. Además, y considerando que una adecuada planeación, seguimiento y control es factor clave en el aumento de éxito de los proyectos en este sector, el $48,9 \%$ no hace uso de metodologías, prácticas o aplica guías para la gerencia de proyectos. Parte de esta falta de aplicación podría ser explicada por la falta de investigaciones acerca de la aplicación de buenas prácticas en pymes del sector de la construcción de edificaciones en Bogotá, en particular en los grupos de procesos de iniciación y planeación. Aunque el PMI refleja gran parte de las prácticas aplicadas en el sector en su extensión para la construcción, se evidencia falta de aplicación de una metodología para la gerencia de proyectos en las pymes. En Bogotá, la investigación en gerencia de proyectos es poca, quizá por falta de cultura en gerencia de proyectos alineada a estándares, guías o metodologías establecidas.

En cuanto a las organizaciones, existen tres perfiles de negocio: a) organizaciones con estrategia de negocio para proyectos de contratación pública, b) estrategia de negocio para proyectos de contratación privada y c) estrategia de negocio para proyectos de inversión; esta última se divide en venta y arriendo dependiendo de los objetivos organizacionales. Encontrar variables unificadas para las tres estrategias de negocio es difícil, debido a las diferencias entre ellas, en especial durante la planificación. Sin embargo, la contratación pública y privada puede llegar a tener similitudes en la gestión de procesos de la gerencia de proyectos, aunque el perfil de inversión sea totalmente diferente en su concepción, planificación y ejecución. Por ejemplo, en cuanto a las similitudes, la definición de alcance del proyecto a nivel público se realiza mediante legalización de contrato $\mathrm{u}$ orden de servicio, que debe ser revisada con el fin de minimizar desviaciones y dar a conocer alcances no especificados al ente contratante. En cuanto a las diferencias, el gerente de proyectos no cuenta con un perfil definido. En algunas organizaciones, la función la cumple el propietario de la empresa, en otras el gerente general y en ocasiones algún socio del proyecto, con ausencia de funciones y responsabilidades claramente definidas para la gerencia del proyecto, lo que puede ser explicado por el tamaño de las organizaciones. Es frecuente encontrar que las organizaciones públicas y privadas en general son pequeñas en comparación con las de inversión, que en muchos casos son medianas acercándose a la gran empresa; por tanto, la robustez organizacional, como la solidez económica y la magnitud de proyectos, difiere una de las otras. Debido a lo anterior, las pymes de pequeño tamaño tienen una menor gestión en el desarrollo de procesos gerenciales alineados a metodologías o guías establecidas, no obstante, son organizaciones que tienen una estructura de gestión de proyectos basados en la experiencia, el conocimiento del negocio y el comportamiento del mercado, lo que permite aplicar mejores prácticas de gerencia moderna. Mientras que la pequeña empresa implementa procesos basados en la experiencia, la mediana empresa, al tener una mayor robustez organizacional y económica, está más alineada a realizar procesos basados en gestión de la calidad, implementación de guías o normas actualmente aceptadas en la 
industria de la construcción edificatoria, así como mejores prácticas en gerencia de proyectos. Se resalta el interés en la pyme de implementar un estándar o guía en la gerencia de los proyectos, para ser aplicados en la gestión de sus procesos; sin embargo, es prudente que las organizaciones traten de subsanar o tengan presente los siguientes aspectos: limitaciones de tiempos, presupuestos ajustados, volatilidad económica, fluctuación del mercado, inestabilidad jurídica, normativa y política.

De acuerdo con el análisis cualitativo realizado a las entrevistas, las debilidades o fortalezas en la gestión de áreas de conocimiento y grupos de procesos para la gerencia de proyectos en las pymes de Bogotá son:

- Identificación, planificación y documentación de los riesgos, seguido de la planificación de las comunicaciones y finalmente la identificación y planificación de los interesados.

- Las organizaciones de carácter privado realizan una mejor gestión en la identificación de interesados, ya que para el desarrollo del proyecto se deben contemplar aspectos normativos, jurídicos, políticos, económicos y ambientales, sin embargo, estas no tienen cultura en la planificación y el registro de lecciones aprendidas.

- En general, las pymes solo asumen riesgos asociados a la gestión de seguridad laboral y riesgo profesional, de igual manera asocian el riesgo al imprevisto, que contemplan dentro del AIU y bajo la experiencia del gerente de proyecto o quien realice estas funciones.
- Las áreas en las que se genera mejor gestión por parte de las organizaciones son la estimación del tiempo y costo, seguido de la planificación de las adquisiciones. En cuanto a la gestión de calidad, en la mayoría de las organizaciones, se visualiza como un gasto y no como una inversión. Es frecuente no encontrar un área encargada de este proceso y es común que el funcionario ejecutor del proyecto sea quien realice la gestión de calidad. En cuanto a los procesos de monitoreo y control de proyectos, en la pequeña empresa, estos se presentan en menor medida que en la mediana empresa, debido a la robustez organizacional y músculo económico; no obstante, es una necesidad inherente desarrollar estructuras fuertes de monitoreo y control a los proyectos.

- La mediana empresa, que comúnmente tiene el perfil de inversión, realiza una mejor gestión en la identificación de interesados, ya que para el desarrollo del proyecto se deben contemplar aspectos, normativos, jurídicos, políticos, económicos y ambientales; sin embargo, no tiene cultura en la planificación y el registro de lecciones aprendidas.

- La definición de criterios de aceptación y éxito de los proyectos depende del tipo de perfil de negocio, cultura empresarial, objetivos estratégicos y visión organizacional.

Debido a lo anterior, se propone realizar un mayor esfuerzo en las siguientes variables clave (Tabla 4), para la futura conformación de un modelo para la gerencia de proyectos en las pymes del sector de la construcción. 
Tabla 4. Variables claves para un modelo futuro de gerencia de proyectos en pymes del sector de la construcción

\begin{tabular}{|c|c|c|c|c|}
\hline Proceso & $\begin{array}{l}\text { Área de conocimiento } \\
\text { o grupo de procesos }\end{array}$ & Pública & Privada & Inversión \\
\hline \multirow[b]{2}{*}{ Iniciación } & \multirow[b]{2}{*}{ Integración } & $\begin{array}{l}\text { Se puede reemplazar el acta de } \\
\text { constitución por los contratos y } \\
\text { las órdenes de servicio, donde en } \\
\text { los anexos se defina claramente el } \\
\text { alcance y las restricciones. }\end{array}$ & \multicolumn{2}{|c|}{$\begin{array}{l}\text { El proyecto debe ser oficializado desde } \\
\text { su nacimiento mediante un documento } \\
\text { formal. }\end{array}$} \\
\hline & & \multicolumn{3}{|c|}{$\begin{array}{l}\text { Desde el nacimiento del proyecto mínimo se debe: } \\
\text { - Estipular los tiempos en fases de planeación y ejecución del proyecto. } \\
\text { - Definir cómo se controlarán las diferentes fases. } \\
\text { - Cómo será el cierre del proyecto. } \\
\text { Esta planeación se puede realizar mediante una reunión kickoff documentada } \\
\text { mediante acta, definiendo tareas y responsables. }\end{array}$} \\
\hline \multirow[t]{2}{*}{ Planeación } & \multirow[t]{2}{*}{ Alcance } & $\begin{array}{l}\text { Solicitar información previa } \\
\text { como diseños, estudios técnicos, } \\
\text { estudio de costos y estudios } \\
\text { socioeconómicos. Si no los hay, } \\
\text { debe quedar registrado. }\end{array}$ & $\begin{array}{c}\text { Revisar los } \\
\text { alcances } \\
\text { establecidos por el } \\
\text { cliente y mediante } \\
\text { reunión clarificar } \\
\text { el alcance y las } \\
\text { restricciones, } \\
\text { dejando } \\
\text { documentados } \\
\text { los cambios o las } \\
\text { adiciones. }\end{array}$ & $\begin{array}{c}\text { Usar una guía } \\
\text { estandarizada } \\
\text { para la definición } \\
\text { del alcance, } \\
\text { dependiendo } \\
\text { del proyecto } \\
\text { por realizar, que } \\
\text { contenga una lista } \\
\text { de chequeo de } \\
\text { los criterios del } \\
\text { alcance. }\end{array}$ \\
\hline & & $\begin{array}{l}\text { Revisar los alcances establecidos } \\
\text { y dejar registradas deficiencias y } \\
\text { fortalezas, así como registrar las } \\
\text { restricciones que se tendrían para } \\
\text { el correcto desarrollo del proyecto. }\end{array}$ & \multicolumn{2}{|c|}{$\begin{array}{l}\text { Establecer el alcance tomando como } \\
\text { base el desglose de actividades. }\end{array}$} \\
\hline \multirow{4}{*}{ Planeación } & \multirow{4}{*}{ Tiempo } & $\begin{array}{l}\text { Revisar los alcances del proyecto, } \\
\text { definiendo tiempos para cada } \\
\text { actividad e identificar si la entidad } \\
\text { contratante contemplo los riesgos } \\
\text { del proyecto, registrar y que haga } \\
\text { parte del contrato. }\end{array}$ & $\begin{array}{l}\text { Definir tiempos } \\
\text { en fases del } \\
\text { proyecto. }\end{array}$ & \\
\hline & & $\begin{array}{l}\text { Establecer reservas de tiempo y } \\
\text { dejar registro en el contrato. }\end{array}$ & \multicolumn{2}{|c|}{$\begin{array}{l}\text { Revisar los alcances del proyecto y } \\
\text { asignar tiempos a cada actividad. }\end{array}$} \\
\hline & & $\begin{array}{l}\text { Planificar bien el flujo de caja y } \\
\text { garantizar que este se materialice. }\end{array}$ & \multicolumn{2}{|c|}{$\begin{array}{l}\text { Identificar los riegos y los tiempos en } \\
\text { caso de materializarse. }\end{array}$} \\
\hline & & & \multicolumn{2}{|c|}{ Establecer reservas de tiempo. } \\
\hline
\end{tabular}


Tabla 4. Variables claves para un modelo futuro de gerencia de proyectos en pymes del sector de la construcción (Continuación)

\begin{tabular}{|c|c|c|c|}
\hline Proceso & $\begin{array}{l}\text { Área de conocimiento } \\
\text { o grupo de procesos }\end{array}$ & Pública & Inversión \\
\hline \multirow{17}{*}{ Planeación } & \multirow[t]{3}{*}{ Costos } & $\begin{array}{l}\text { Revisar el alcance conjuntamente } \\
\text { con los tiempos establecidos y } \\
\text { hacer un análisis de los costos, } \\
\text { identificando si la entidad } \\
\text { contratante contempló los riesgos } \\
\text { del proyecto, registrar el resultado } \\
\text { y que haga parte del contrato. }\end{array}$ & $\begin{array}{l}\text { Revisar el alcance conjuntamente con } \\
\text { los tiempos establecidos y hacer un } \\
\text { análisis de los costos. }\end{array}$ \\
\hline & & $\begin{array}{l}\text { Establecer reservas en costos y } \\
\text { dejar registro en el contrato. }\end{array}$ & $\begin{array}{l}\text { Identificar los riegos y los costos en } \\
\text { caso de materializarse. }\end{array}$ \\
\hline & & $\begin{array}{l}\text { Planificar bien el flujo de caja y } \\
\text { garantizar que este se materialice. }\end{array}$ & $\begin{array}{l}\text { Establecer costos por desglose de } \\
\text { actividades y los APUS. }\end{array}$ \\
\hline & \multirow{5}{*}{ Calidad } & \multicolumn{2}{|c|}{$\begin{array}{l}\text { Planificar la gestión de la calidad mediante un enfoque en calidad de recursos } \\
\text { humanos y contrataciones. }\end{array}$} \\
\hline & & \multicolumn{2}{|c|}{$\begin{array}{c}\text { Garantizar el cumplimiento de las normas reguladoras en la construcción de } \\
\text { edificaciones. }\end{array}$} \\
\hline & & \multicolumn{2}{|c|}{$\begin{array}{l}\text { Definir seguimientos a las actividades mediante auditorías internas del } \\
\text { personal más experimentado. }\end{array}$} \\
\hline & & \multicolumn{2}{|c|}{$\begin{array}{l}\text { Establecer desde el nacimiento del proyecto los controles claves de calidad } \\
\text { mediante una lista de chequeo. }\end{array}$} \\
\hline & & \multicolumn{2}{|c|}{$\begin{array}{c}\text { En lo posible, realizar procesos de certificación con entidades externas o en } \\
\text { su defecto establecer una estructura interna de calidad, mediante auditorías y } \\
\text { visitas de obra. }\end{array}$} \\
\hline & \multirow{6}{*}{ Recursos humanos } & $\begin{array}{l}\text { Definir los perfiles de cargo para la } \\
\text { ejecución del proyecto. }\end{array}$ & $\begin{array}{l}\text { La gestión del recurso humano se debe } \\
\text { hacer desde el nacimiento del proyecto. }\end{array}$ \\
\hline & & $\begin{array}{l}\text { Establecer la necesidad de recurso } \\
\text { humano basado en la planeación } \\
\text { del proyecto. }\end{array}$ & $\begin{array}{l}\text { Definir los perfiles de cargo para cada } \\
\text { fase del proyecto. }\end{array}$ \\
\hline & & $\begin{array}{l}\text { Contar con bases de datos de } \\
\text { personal especializado en el sector. }\end{array}$ & $\begin{array}{l}\text { Establecer la necesidad de recurso } \\
\text { humano basado en las fases del } \\
\text { proyecto. }\end{array}$ \\
\hline & & $\begin{array}{l}\text { Diseñar estrategias que ayuden a } \\
\text { minimizar la rotación del personal. }\end{array}$ & $\begin{array}{l}\text { Contar con bases de datos de personal } \\
\text { especializado en el sector. }\end{array}$ \\
\hline & & $\begin{array}{l}\text { Diseñar estrategias para mejorar } \\
\text { los ambientes laborales. }\end{array}$ & $\begin{array}{l}\text { Diseñar estrategias que ayuden a } \\
\text { minimizar la rotación del personal. }\end{array}$ \\
\hline & & & $\begin{array}{l}\text { Diseñar estrategias para mejorar los } \\
\text { ambientes laborales. }\end{array}$ \\
\hline & \multirow{3}{*}{ Comunicaciones } & \multicolumn{2}{|c|}{ Definir un plan de comunicación desde el nacimiento del proyecto. } \\
\hline & & \multicolumn{2}{|c|}{$\begin{array}{l}\text { Establecer los perfiles que generen liderazgo y contemplar estrategias de } \\
\text { comunicación efectiva. }\end{array}$} \\
\hline & & \multicolumn{2}{|c|}{$\begin{array}{c}\text { Informar a todo el equipo de trabajo los objetivos del proyecto, alcances, } \\
\text { beneficios y avances del proyecto. }\end{array}$} \\
\hline
\end{tabular}


Tabla 4. Variables claves para un modelo futuro de gerencia de proyectos en pymes del sector de la construcción (Continuación)

\begin{tabular}{|c|c|c|c|c|}
\hline Proceso & $\begin{array}{l}\text { Área de conocimiento } \\
\text { o grupo de procesos }\end{array}$ & Pública & Privada & Inversión \\
\hline \multirow{18}{*}{ Planeación } & \multirow{3}{*}{ Riesgos } & \multicolumn{3}{|c|}{$\begin{array}{c}\text { Los riesgos deben ser identificados, planificados, evaluados, registrados y } \\
\text { divulgados a todo el equipo de trabajo. }\end{array}$} \\
\hline & & \multicolumn{3}{|c|}{$\begin{array}{c}\text { La gestión de riesgos debe hacerla el personal que cuente con el know-how de } \\
\text { la organización. }\end{array}$} \\
\hline & & \multicolumn{3}{|c|}{ Elaborar un registro de riesgos. } \\
\hline & \multirow{8}{*}{ Adquisiciones } & \multicolumn{3}{|c|}{$\begin{array}{l}\text { Establecer contratos y servicios, en los mismos términos que la organización } \\
\text { los haya firmado con el contratante. }\end{array}$} \\
\hline & & \multicolumn{3}{|c|}{ Contar con una base de datos claves de proveedores. } \\
\hline & & \multicolumn{3}{|c|}{$\begin{array}{c}\text { Realizar programación de compras y contratos, basado en los tiempos del } \\
\text { cronograma del proyecto. }\end{array}$} \\
\hline & & \multicolumn{3}{|c|}{$\begin{array}{l}\text { Planificar el flujo de caja para las adquisiciones y garantizar que este se } \\
\text { materialice. }\end{array}$} \\
\hline & & \multicolumn{3}{|c|}{ Identificar riesgos por la dinámica económica del país. } \\
\hline & & \multicolumn{3}{|c|}{ Las adquisiciones deben ser manejadas por personal de absoluta confianza. } \\
\hline & & & $\begin{array}{l}\text { La planifica } \\
\text { deberá realiz }\end{array}$ & $\begin{array}{l}\text { lquisiciones se } \\
\text { ase de punto de } \\
\text { o. }\end{array}$ \\
\hline & & & Todas las a & $\begin{array}{l}\text { nes deben ser } \\
\text { las. }\end{array}$ \\
\hline & \multirow{3}{*}{ Interesados } & $\begin{array}{c}\text { Definir con la entidad contratante } \\
\text { quién es el encargado de la } \\
\text { identificación, caracterización } \\
\text { y relacionamiento con los } \\
\text { stakeholders. }\end{array}$ & $\begin{array}{l}\text { Identificar los } \\
\text { correcto d }\end{array}$ & $\begin{array}{l}\text { los claves para el } \\
\text { del proyecto. }\end{array}$ \\
\hline & & $\begin{array}{l}\text { Dejar registro de quien asume } \\
\text { los contratiempos que se puedan } \\
\text { presentar por la gestión de } \\
\text { interesados. }\end{array}$ & $\begin{array}{r}\text { Establ } \\
\text { relacionami }\end{array}$ & $\begin{array}{l}\text { tegias de } \\
\text { os interesados. }\end{array}$ \\
\hline & & & $\begin{array}{l}\text { La identifica } \\
\text { relacionami }\end{array}$ & $\begin{array}{l}\text { teresados y su } \\
\text { be ser realista. }\end{array}$ \\
\hline & \multirow{4}{*}{ Seguimiento y control } & \multicolumn{3}{|c|}{$\begin{array}{c}\text { Definir claros procedimientos en controles de cambio. Solicitar doble } \\
\text { verificación a controles de cambio, con registro de quién solicita el cambio y } \\
\text { quién lo autoriza. }\end{array}$} \\
\hline & & & Los cambios & $\begin{array}{l}\text { concertados con } \\
\text { e. }\end{array}$ \\
\hline & & & $\begin{array}{l}\text { Desde el ini } \\
\text { debe ser in } \\
\text { desviaciones } \\
\text { plazos por }\end{array}$ & $\begin{array}{l}\text { yecto el cliente } \\
\text { obre posibles } \\
\text { ampliación de } \\
\text { ue se soliciten. }\end{array}$ \\
\hline & & \multicolumn{3}{|c|}{$\begin{array}{l}\text { Mediante un procedimiento de lecciones aprendidas se debe garantizar la } \\
\text { transferencia de conocimiento entre proyectos o tipos de negocio. }\end{array}$} \\
\hline
\end{tabular}

Fuente. Elaboración propia. 


\section{Conclusiones}

E $n$ este artículo, se presentó el diagnóstico - de prácticas de iniciación y planeación en gerencia de proyectos en pymes del sector de la construcción edificatoria en Bogotá, mediante una revisión bibliográfica de 181 documentos académicos, de los cuales 80 fueron de lectura obligatoria. En la investigación, que se clasifica como cualitativa con alcance exploratorio, se aplican 18 entrevistas semiestructuradas a profundidad y 92 encuestas estructuras cerradas a gerentes de proyectos o los que hagan su función en pymes del sector de la construcción en Bogotá.

Entre las prácticas encontradas, se destaca una adecuada gestión en los procesos de tiempo, costos, adquisiciones y compras. Se identificó la necesidad de generar fortalecimiento y mejoras en los procesos de calidad, gestión humana y comunicaciones. Por otra parte, se halló que los procesos de identificación, análisis y evaluación de riesgos e interesados presentan poca o nula gestión en la gerencia de proyectos, al igual que desconocimiento para su aplicación en la pequeña y mediana empresa.

Se evidencia que en Bogotá la investigación en gerencia de proyectos es poca, quizá por falta de cultura en gestión de proyectos alineada a estándares, guías o metodologías establecidos. Se concluye que la experiencia, el conocimiento del mercado y la estructura organizacional son la base para la gerencia de proyectos. De igual manera, buena cantidad de las prácticas encontradas en las entrevistas se reflejan en la extensión para la construcción del PMBOK, pero esto no significa que las pymes apliquen una metodología para la gerencia de proyectos. Por tal razón, se identifica la necesidad de mejorar los procesos en la gerencia de proyectos para pymes de construcción edificatoria.

\section{Recomendaciones y trabajo futuro}

Se recomienda a la pyme analizar e - implementar los resultados obtenidos según las variables expuestas con anterioridad (Tablas 1, 2 y 3). Vale la pena mencionar que estas variables aplicarían para empresas que estén en el sector de la construcción edificatoria y para que la estrategia de negocio cumpla con las halladas en la investigación.

Se recomienda a la mediana empresa hacer un buen estudio de mercado, en el que se reflejen el potencial y la prefactibilidad del producto que se va a desarrollar, en comparación con la pequeña, la cual en ocasiones desarrolla proyectos de inversión y donde este estudio se ve reducido a limitaciones económicas.
Se recomienda a la pyme optimizar los procesos de planeación, basados en la mejora continua en la gestión del tiempo, costo y adquisiciones. De igual manera, tomar acciones correctivas y de fortalecimiento a los procesos de calidad, recursos humanos y comunicaciones. Finalmente, empezar a implementar los procesos de identificación, análisis y evaluación de riesgos e interesados.

Introducir la extensión del PMBOK para el sector de la construcción en la gerencia del proyecto puede aportar al inicio de la aplicación de metodologías formales para realizar la gerencia de proyectos, ya que esta 
extensión recopila algunas de las buenas prácticas identificadas durante la presente investigación.

Hoy en día, el sector de la construcción lleva a cabo proyectos en los cuales la iniciación y la planificación del proyecto se hacen en paralelo con la ejecución, el control y el cierre del proyecto, denominados fast-track; en este sentido, se pueden desarrollar investigaciones sobre la gerencia de proyectos para esta nueva denominación de proyectos. De igual manera, reconociendo las limitaciones culturales y geográficas de esta investigación, es importante poder extenderla a otras regiones del país, tipo de organizaciones, así como a otros sectores económicos. También, realizar un comparativo con la gerencia de proyectos en grandes empresas.

\section{Referencias}

Ahlemann, F., El Arbi, F., Kaiser, M. G. y Heck, A. (2013). A process framework for theoretically grounded prescriptive research in the project management field. International Journal of Project Management, 31 (1), 43-56. http://doi.org/10.1016/j.ijproman.2012.03.008

Andersen, H., Cobbold, I. y Lawrie, G. (2001). Balanced scorecard implementation in SMEs: Reflection on literature and practice. Ponencia presentada en 4th SME International Conference, Allborg University, Dinamarca.

Aragón Sánchez, A. y Rubio Bañón, A. (2005). Factores asociados con el éxito competitivo de las pymes industriales en España. Universia Business Review, 8, 38-51.

Baumol, W. J. (2002). The free-market innovation machine: Analyzing the growth miracle of capitalism. Princeton: Princeton University Press.

López, B. (2006). La búsqueda bibliográfica: componente clave del proceso de investigación. Diaeta, 24(115), 31-37.

Diez-Silva, H. M., Pérez Ezcurdia, M. A. y Gimena Ramos, F. N. (2012). Medición del desempeño y éxito en la dirección perspectiva del manager público. Revista EAN, 73, 60-79.

Dvir, D., Raz, T. y Shenhar, A. J. (2003). An empirical analysis of the relationship between project planning and project success. International Journal of Project Management, 21(2), 89-95. http://doi.org/10.1016/ S0263-7863(02)00012-1.

Elonen, S. y Artto, K. A. (2003). Problems in managing internal development projects in multi-project environments. International Journal of Project Management, 21(6), 395-402. http://doi.org/10.1016/ S0263-7863(02)00097-2.
Faniran, O. O., Oluwoye, J. O. y Lenard, D. (1994). Effective construction planning. Construction Management and Economics, 12(6), 485-499. http:// doi.org/10.1080/01446199400000060.

Geroski, P. y Machin, S. (1992). Do innovating firms outperform non—innovators? Business Strategy Review, 3(2), 79-90. http://doi.org/10.1111/j.1467-8616.1992. tb00030.x

Ghobadian, A. y Gallear, D. N. (1996). Total quality management in SMEs. The International Journal of Management Science, 24(81), 3-106. http://doi. org/10.1016/0305-0483(95)00055-0.

Ghobadian, A. y Gallear, D. (1997). TQM and organization size. International Journal of Operations $\mathcal{E}$ Production Management, 17(2), 121-163. http://doi. org/10.1108/01443579710158023.

González, J. A., Solís, R. y Alcudia, C. (2010). Diagnóstico sobre la planeación y control de proyectos en las pymes de construcción. Revista de la Construcción, 9(1), 17-25. http://doi.org/10.4067/S0718-915X2010000100003

Haponava, T. y Al凹ibouri, S. (2009). Identifying key performance indicators for use in control of pre $\square$ project stage process in construction. International Journal of Productivity and Performance Management, 58(2), 160-173. http://doi.org/10.1108/17410400910928743

Hernández Sampieri, R., Fernández Collado, C. y Baptista Lucio, P. (2010). Metodología de la investigación (5. ${ }^{\mathrm{a}}$ ed.). México: McGraw-Hill.

Hernández Sampieri, R., Fernández Collado, C. y Baptista Lucio, P. (2014). Metodología de la investigación (6. a ed.). México: McGraw-Hill. 
Heunks, F. J. (1998). Innovation, creativity and success. Small Business Economics, 10(3), 263-272. http://doi. org/10.1023/A:1007968217565

Iglesias, M. G., Somohano, F. M., Rosario, A. H. De Papis, L. C. y Rodrigues, L. L. (2012). Factores explicativos de la implantación de los ERP en las pymes: el caso de Cantabria. Revista Internacional de la Pequeña y Mediana Empresa, 1(4), 1-28.

Lehtonen, P. y Martinsuo, M. (2008). Change program initiation: Defining and managing the program-organization boundary. International Journal of Project Management, 26(1), 21-29. http://doi.org/10.1016/j. ijproman.2007.07.003.

Lo, V. H. Y. y Humphreys, P. (2000). Project management benchmarks for SMEs implementing ISO 9000. Benchmarking: An International Journal, 7(4), 247-259. http://doi.org/10.1108/14635770010378891.

Madrid Guijarro, A. y García Pérez de Lema, D. (2008). Las ayudas financieras a la innovación a la pyme: sesgo de motivación y de selección administrativa. Revista Internacional de la Pequeña y Mediana Empresa, 1(1), 17-35.

Marcella, M. y Rowley, S. (2014). An exploration of the extent to which project management tools and techniques can be applied across creative industries through a study of their application in the fashion industry in the North East of Scotland. International Journal of Project Management, 33(4), 735-746. http:// doi.org/10.1016/j.ijproman.2014.12.002.

Martinsuo, M. y Lehtonen, P. (2007). Program and its initiation in practice: Development program initiation in a public consortium. International Journal of Project Management, 25(4), 337-345. http://doi.org/10.1016/j. ijproman.2007.01.011.

Meister, W. (2006).. Successful project management for small to medium enterprises (SMEs). Ponencia presentada en PMI ${ }^{\circledR}$ Global Congress 2006-Asia Pacific, Bangkok, Thailandia.

Meskendahl, S. (2010). The influence of business strategy on project portfolio management and its success: A conceptual framework. International Journal of Project Management, 28(8), 807-817. http://doi.org/10.1016/j. ijproman.2010.06.007

Morris, P. W. G. y Pinto, J. K. (eds.). (2004). The Wiley Guide to Managing Projects. Hoboken: John Wiley \& Sons, Inc. http://doi.org/10.1002/9780470172391

Murphy, A. y Ledwith, A. (2007). Project management tools and techniques in high $\square$ technology SMEs. Management Research News, 30(2), 153-166. http:// doi.org/10.1108/01409170710722973.

Novak, J. (1991). Clarify with concept maps. The Science Teacher, 58(7).
Platje, A., Seidel, H. y Wadman, S. (1994). Project and portfolio planning cycle. International Journal of Project Management, 12(2), 100-106. http://doi. org/10.1016/0263-7863(94)90016-7

Quesado Rodríguez, P., Aibar Guzmán, B. y Lima Rodríguez, L. (1989). El cuadro de mando integral como herramienta de gestión estratégica en pequeñas y medianas empresas portuguesas, Revista Internacional de la Pequeña y Mediana Empresa, 1 (4), 90-128.

Project Management Institute - PMI (2013). A guide to the project management body of knowledge (PMBOK® guide) - Fifth edition. Project Management Institute, Newtown Square, PA.

Porter, M. E. (1908). Competitive strategy: Techniques for analyzing industries and competitors. Nueva York: The Free Press.

Rahman, S. (2001). A comparative study of TQM practice and organisational performance of SMEs with and without ISO 9000 certification. International Journal of Quality \& Reliability Management, 18(1), 35-49. http:// doi.org/10.1108/02656710110364486.

Romero Espinosa, F., Melgarejo Molina, Z. A. y Vera-Colina, M. A. (2015). Fracaso empresarial de las pequeñas y medianas empresas (pymes) en Colombia. Suma de Negocios, 6(13), 29-41. http://doi.org/10.1016/j.sumneg.2015.08.003

Rubiano Ovalle, Ó. y Cuadros López, Á. J. (2012). Estado de la gerencia de proyectos en pymes del sector artes gráficasy propuestas de lineamientos para su mejoramiento. Sotavento MBA, 19, 16-30.

Sdrolias, L., Sirakoulis, K., Trivellas, P. y Poulios, T. (2005). Applicability of project management techniques in SMEs: Evidence from Greece. Studia Universitatis Babes-Bolyai, 8(1), 44-52.

Son, J., \& Rojas, E. M. (2010). Impact of optimism bias regarding organizational dynamics on project planning and control. Journal of Construction Engineering and Management, 137 (2), 147-157. http://doi.org/10 .1061/ $\square$ ASCE๑CO.1943-7862.0000260.

Tannock, J., Krasachol, L. y Ruangpermpool, S. (2002). The development of total quality management in Thai manufacturing SMEs: A case study approach. International Journal of Quality \& Reliability Management, 19(4), 380-395. http://doi.org/10.1108/02656710210421562.

Unit, D. (2014). A survey-based study of project management problems in small and medium scale enterprises (SMEs) in Nigeria. European Scientific Journal, 10(25), 40-57.

Varajão, J., Domínguez, C., Ribeiro, P. y Paiva, A. (2014). Critical success aspects in project management: Similarities and differences between the construction and the software industry. Tehnicki Vjesnik / Technical Gazette, 21(3), 583-589. 
Vargas Zambrano, J. C. (2015). Análisis sector construcción en Colombia. Recuperado de http://www.pmicolombia. org/2015/08/analisis-sector-construccion-en-colombia/

Vega Rodríguez, R. A., Castaño Ramírez, A. y Mora Ramírez, A. J. (2011). Pymes: reflexiones para la pequeña y mediana empresa en Colombia. Bogotá: Politécnico Grancolombiano.

Verbano, C. y Venturini, K. (2013). Managing risks in SMEs: A literature review and research agenda. Journal of Technology Management E Innovation, 8(3), 186-197.

Warszawski, A. (1984). Planning and organization of research in building. Engineering, 109(3), 261-275. http:// doi.org/10.1061/(ASCE)0733-9364(1983)109:3(261). 
